\title{
Capacidad intelectual y función ejecutiva en niños intelectualmente talentosos y en niños con inteligencia promedio*
}

\author{
Intellectual Quotient and Executive Function \\ in Gifted and Average IQ Children
}

Recibido: septiembre 22 de 2009 ～Revisado: enero 12 de 2010 Aceptado: febrero 3 de 2010

\author{
David A Montoya-Arenas ** \\ Universidad San Buenaventura, Medellín, Colombia \\ Natalia Trujillo-OrRego *** \\ DAVID A. PINEDA-SALAZAR ${ }^{* * * *}$ \\ Universidad de Antioquia, Medellín, Colombia
}

Para citar este artículo. Montoya-Arenas, D.A., Trujillo-Orrego, N. \& Pineda-Salazar, D. (2010). Capacidad intelectual y función ejecutiva en niños intelectualmente talentosos y en niños con inteligencia promedio. Universitas Psychologica, 9 (3), 737-747.

* Artículo de investigación

** Grupo de Neuropsicología y Conducta-GRUNECO, Facultad de Psicología, Universidad de San Buenaventura, Medellín, Colombia. E-mail: davidmontoya-@hotmail.com.

*** GRUNECO. Calle 62 \# 52-59 Lab. 412. SIU. Universidad de Antioquia, Medellín, Colombia. Correo electrónico: natalitra@hotmail.com

${ }^{* * * * *}$ GRUNECO. Calle 62 \# 52-59 Lab. 412. SIU. Universidad de Antioquia, Medellín, Colombia. Doctorado en ciencias básicas biomédicas, Facultad de Medicina, Universidad de Antioquia. E-mail: dpineda@epm.net.co

\section{RESUMEN}

El objetivo del presente estudio fue analizar las relaciones, por comparación y correlación, entre las dimensiones de función ejecutiva (FE) y capacidad intelectual (CI) en niños con talento excepcional (NTE) y niños con inteligencia promedio (NIP), de 7 a 11 años de edad, de la ciudad de Medellín, Colombia. Se seleccionó una muestra no aleatoria de 62 niños y se conformaron dos grupos independientes, de acuerdo con la CI. Se les administraron pruebas de FE. Según los resultados, los NTE tuvieron una ejecución significativamente más alta en la TFV-fonológica $(p<0,05)$, con un tamaño del efecto medio $(0,65)$. Únicamente, se encontró una correlación estadísticamente significativa pero baja $(r=0,267, p=0,03)$ entre TFV-fonológica y el CIV. Se concluyó que la FE parece ser una actividad cognitiva no relacionada con las mediciones de la CI.

Palabras clave autores

Capacidad intelectual, función ejecutiva, fluidez verbal, talentos, superdotación. Palabras clave descriptor

Niños (7-11 años), aspectos psicológicos, pruebas de inteligencia, función ejecutiva.

\footnotetext{
A B S T R A C T

Objective: To analyze the relationship, by comparison and correlation, between executive function (EF) and intellectual capacity (IC) in clever children (CC) and Average Intelligence Quotient children (AIQC), aged 7 to 11 years, and living in Medellín - Colombia. Patients and Methods: a non-randomized sample of 62 children was selected. The sample was constituted by two independent groups, according the IC. EF tasks were administered to both groups. Results: CC had statistically significant higher performance on phonological verbal fluency test (PVFT) $(\mathrm{p}<0,05)$, with a modest effect size $(0,65)$. A statistically significant, but low $(r=0,267, p$ $=0,03)$, correlation between PVF and verbal IQ was found. Conclusion: EF appeared as a non IQ-related ability.

Keywords authors Intellectual Capacity, Executive Function, Verbal Fluency, Talents, Giftedness. Keywords plus Children (7-11 years), Psychological Aspects, Intelligence Tests, Executive Function.
} 


\section{Introducción}

La capacidad intelectual (CI), como medida de la inteligencia, es quizá uno de los temas de la psicología más estudiados a través del tiempo, por la complejidad del constructo y la poca unificación entre las teorías y modelos que buscan explicar su desarrollo, evaluación y uso (Carrol, 1993; Cattel, 1963). La psicometría tradicional remplazó el concepto de edad mental y construyó una puntuación compuesta y estandarizada de la CI, derivada de las escalas de inteligencia, que se denominó coeficiente intelectual. Dicha perspectiva considera que la evaluación de la inteligencia puede ser dividida en múltiples destrezas de tipo ejecutivo y lingüístico, a las cuales se les da un valor numérico independiente basada en el desempeño del sujeto, el cual al ponderarse da cuenta del coeficiente intelectual (Spearman, 1923).

Desde la perspectiva neuropsicológica, el concepto de CI se ha asumido como un constructo de dimensiones múltiples compuesto por tres estratos: 1) aptitudes o factores específicos: razonamiento, lenguaje, recepción auditiva, producción de ideas, velocidad cognitiva, aptitudes psicomotrices y características personales; 2) aptitudes o factores amplios: inteligencia fluida (son procesos de razonamiento inducción y visualización), inteligencia cristalizada (referida a la capacidad de utilizar su inteligencia general en la adquisición de tipos diversos de conocimiento cultural; implica aptitudes del lenguaje, razonamiento cuantitativo y conocimiento mecánico); aptitud general de memoria y aprendizaje, percepción visual y aptitud viso-espacial; y, 3) Factor G: hace referencia a un factor genérico de inteligencia general (Carrol, 1993, 1994; Guilford, 1985; Thurstone, 1947).

La función ejecutiva (FE), por su parte, se ha asumido desde el modelo factorial como un constructo de múltiples dimensiones o factores, el cual está constituido por tres subsistemas (Stuss, 1992):

- El primer sistema es el de entrada de la información, que se corresponde con el sistema sensorial y perceptual, y que contiene un dominio para cada módulo. En este sistema el análisis y la respuesta perceptual pueden ser simples o complejos, además son el resultado de conductas aprendidas, automáticas e inmediatas.

- El segundo sistema es el comparador, el cual se asocia con el control ejecutivo o supervisión del comportamiento. Neuroanatómicamente las conexiones recíprocas entre las áreas de asociación multimodal retrorrolándicas, el sistema límbico y el cerebro anterior proveen las bases neurales necesarias para este control ejecutivo. Dicho sistema de control se ha dividido conceptual y experimentalmente en subfunciones específicas, que son: la anticipación, la selección de objetivos y la elaboración de planes. Este sistema se activa ante situaciones novedosas, las cuales demandan control cognitivo y supervisión, hasta cuando el sistema consigue automatizarlas.

- El tercer sistema es el de salida, el cual incorpora el concepto de autoconciencia y autorreflexión. Este sistema se relaciona con la capacidad de ser consciente de "sí mismo" y de reflejar en pensamientos y conductas los patrones individuales del yo. La autoconciencia, en este sentido, depende de los inputs que recibe de los sistemas sensorial-perceptual y de control ejecutivo, mientras que el output influye en la naturaleza y el grado del control ejecutivo.

Las investigaciones sobre la correlación de estos dos constructos de la cognición humana son escasas y en su mayoría han sido enfocadas en fenómenos patológicos, o traumáticos (Damasio \& Anderson, 1993; Mark et al., 2002). Sólo 4 de ellas fueron realizadas en muestras de niños y en jóvenes saludables (Ardila, Pineda \& Roselli, 2000; Arffa, 2007; Arffa, Lovell, Podell \& Golberg, 1998; Friedman et al., 2006; Welsh, Pennington \& Groisser, 1991).

Dado el limitado estado del arte en relación con esta temática, en especial en población infantil y juvenil, el trabajo investigativo para establecer los límites conceptuales de cada constructo, los cuales se apoyen en datos empíricos sólidos que permitan derivar modelos neuropsicológicos teóricos para una adecuada evaluación clínica y diagnósticos 
adecuados, adquieren singular importancia. Las investigaciones revisadas hasta la fecha sobre el tema son contradictorias; algunos estudios consideran que la $\mathrm{CI}$ y la FE son actividades cognitivas totalmente separadas (Friedman et al., 2006; Milner, 1982). Otros postulan que son independientes, pero que tienen correlaciones en algunas de sus dimensiones (Donders \& Kirsch, 1991). Mientras que otro grupo supone que, por su naturaleza de actividades de nivel superior en la cognición humana, están altamente correlacionadas (Ardila et al., 2000). Las diferencias en estas posiciones pudieran originarse en la diversidad de los modelos teóricos, en la metodología de medición derivada y en el tipo de análisis de los datos. También se pueden presentar diferencias en las correlaciones provenientes de las características de los grupos estudiados (adultos, niños, escolaridad alta o baja, etc.) (Arffa, 2007).

Uno de los supuestos que se ha debatido, es la ausencia relativa de componentes de la FE en la administración de tests clásicos de inteligencia, lo cual ha sido descrito también por algunos autores (Denckla, 1996; Stuss, 1992; Welsh et al., 1991). A pesar de que se ha considerado que la actividad cognitiva propositiva y organizada (función ejecutiva) forma parte del constructo de capacidad intelectual, de acuerdo con las definiciones de varios autores (Donders \& Kirsch, 1991; Wechsler, 2004). A su vez, sólo unas pocas investigaciones han encontrado que algunas tareas incluidas en las pruebas de inteligencia, estarían comprendidas en los análisis de factores estudiados como componentes de la FE, bajo la designación de inteligencia fluida (Blair, 2006; García-Barrera, 2008)

La ausencia de correlación entre la CI y la FE ya había sido descrita por los análisis clínicos de pacientes lesionados del lóbulo frontal por $\mathrm{Da}$ masio y Anderson (1993), quienes encontraron en los pacientes con lesiones extensas del lóbulo frontal no había alteración en las puntuaciones de las pruebas estandarizadas que midieron la capacidad intelectual general, que se suponen calculan el Factor G. Por eso, algunos autores proponen el concepto de conducta ejecutiva, como un elemento independiente de la FE y de la inteligencia cognitiva (Arango-Tobón \& Puerta-Lopera, 2008; García-Barrera, 2008; Gioia, Isquith, Retzlaff \& Espy, 2002).

Los niños con talento excepcional (NTE), se definen como personas con un rendimiento superior en cualquier área socialmente valiosa de la conducta humana (De Zubiría, 2000) Sin embargo, en la práctica, el concepto de NTE se ha limitado al campo del desempeño académico, especialmente en lenguas, ciencias sociales, ciencias naturales y matemáticas. Recientemente se ha ampliado el concepto a campos artísticos, como la música, artes gráficas y plásticas, artes representativas y mecánicas, y al ámbito de las relaciones humanas (Passow, 1993).

El fenómeno de identificación de los NTE en Colombia es reciente, y ha tenido como fin apoyar sus necesidades educativas especiales y potencializar sus capacidades, bajo modelos que se centran en la estimulación de las FE, con el supuesto de que unas dimensiones de la FE altamente eficientes son el sustento de la excepcionalidad intelectual (Ardila et al., 2000).

Este estudio tuvo como propósito describir la correlación entre las dimensiones de la FE y la CI en una muestra de NTE $(\mathrm{CI} \geq 130)$ y niños con inteligencia promedio (NIP) (CI entre 90 y 110), de 7 a 11 años de edad, que residen en Medellín, Colombia, para dilucidar si la medición de la inteligencia incluye en su varianza una proporción importante de la FE, entendida como la capacidad de resolver problemas cognitivos de manera intencionada y regulada. Si esto es cierto, se supondría que el rendimiento de los NTE en las tareas de FE debería ser significativamente superior al de los NIP. Con los anteriores supuestos, se intentaría sustentar el modelo según el cual una CI excepcional tiene una correlación directa con una FE excepcional (Arffa, 2007).

\section{Método}

\section{Participantes}

La investigación fue de tipo no experimental con una muestra no aleatoria de 62 niños entre los 7 
y 11 años de edad (30 de género femenino y 32 de género masculino), pertenecientes a varias instituciones privadas de la ciudad de Medellín, Colombia. La muestra se conformó por dos grupos seleccionados de manera independiente, de acuerdo con la CI, la cual fue medida con el coeficiente intelectual total (CIT): 1) aquellos con CIT $\geq 130$, los cuales se clasificaron como NTE, de acuerdo con los parámetros de la Ley 115 del ministerio de educación nacional; y, 2) los que tuvieron un CIT entre 90 y 110, constituyeron el grupo de NIP. En ambos se controló que no existieran diagnósticos neurológicos ni psiquiátricos, mediante una entrevista acerca de antecedentes médicos, entre los cuales se indagaron acerca de consultas por epilepsia, trastornos de aprendizaje, trastornos disruptivos de la conducta, trastornos generalizados del desarrollo, depresión, trastornos de ansiedad, etc.

Dado que en Colombia los colegios privados establecen una selección socioeconómica, derivada de la capacidad de pago de las matrículas y las pensiones mensuales, la mayoría de los niños que estudian en estas instituciones son de estratos socioeconómicos medios y altos. Por esta razón, hubo un sesgo de selección de la muestra, lo que llevó a obtener un mayor número de niños de estas condiciones sociales. Las familias de estrato medio, en Colombia, tienen unos ingresos mensuales entre 500 y 2000 dólares al mes. Las familias de estratos altos tienen ingresos superiores a los 3.000 dólares al mes. Todos estos niños tienen acceso a servicios públicos completos, automóviles privados y están familiarizados con el uso de alta tecnología, que incluye el uso habitual de computadores, teléfonos móviles, juegos de video y acceso a la red.

\section{Instrumentos}

Todos los participantes fueron valorados con el WISC-III, instrumento multifactorial, que permite obtener las siguientes dimensiones: inteligencia general (CI Total), Inteligencia Cristalizada (CI Verbal) e Inteligencia Fluida (CI Ejecución), que constituyen el constructo de capacidad Intelectual.
Para la medida de la función ejecutiva, primero se seleccionaron las dimensiones metacognitivas más significativas (inhibición, planificación, fluidez verbal, fluidez no verbal, monitoreo y selección de objetivos) y, luego, se utilizó, para cada una de ellas, los siguientes instrumentos, respectivamente:

\section{Stroop test}

La versión que se utilizó consta de tres láminas, y cada una contiene 100 elementos distribuidos en cinco columnas de 20 elementos. La primera lámina la forman las palabras 'ROJO', 'VERDE' y 'AZUL', organizadas al azar e impresas en tinta negra. La segunda lámina consiste en 100 elementos idénticos ('XXXX') impresos en tinta azul, verde o roja. En la tercera lámina, aparecen las palabras de la primera lámina impresas en los colores de la segunda, mezcladas ítem por ítem; el primer ítem es el color del ítem 1 de la primera lámina impreso en la tinta del color del ítem 1 de la segunda lámina. No coincide en ningún caso el color de la tinta con el significado de la palabra. Puede ser, por ejemplo, la palabra VERDE impresa en color rojo. El sujeto debe nombrar el color de la tinta. Es aplicable de los 7 a los 80 años (Golden, 1981). Utilizado en población colombiana (Ardila \& Roselli, 1991; Pineda, 1996).

\section{Torre de Hanoi}

Es una prueba de organización, planificación y previsión de conductas, flexibilidad cognitiva, programación viso-espacial de una secuencia de movimientos y de memoria operativa. Tiene diferentes versiones, pero en todas se utilizan anillos de distintos colores y tamaños, y la instrucción es la misma, trasladar las piezas del lado izquierdo al derecho, una por una, hasta lograr reproducir correctamente el modelo representado gráficamente, utilizando las siguientes reglas: Sólo puede coger los anillos de uno en uno y cuando saque uno debe introducirlo en otro poste; Siempre que coloque un anillo encima de otro el que se sitúe encima deberá ser menor que el de debajo y realizarlo en el menor 
número de movimientos que le sea posible. En la prueba se valora el tiempo empleado, el número de movimientos realizados y el tipo de errores cometidos (Álvarez, 2006).

El test de fluidez verbal: fonológico - $|\mathrm{f} /,| \mathrm{a} /, / \mathrm{s} /$ - , y semántico - animales y frutas -

Se mide mediante el número de palabras producidas dentro de cada categoría en un minuto, y es considerado una prueba de producción verbal controlada y programada, que es sensible a las alteraciones en el funcionamiento de las áreas prefrontales izquierdas; instrumento que ha sido estandarizado para población colombiana (Ardila, Rosselli \& Puente, 1994).

\section{Test de Fluidez de diseños (RUFFT)}

Es una prueba de fluidez no verbal, análoga a la prueba (FAS), la prueba está dividida en cinco tareas diferentes: lo común a ellas es hallar una hoja que se encuentra dividida en 35 cuadrados y en cada uno de ellos se encuentran cinco puntos. En la primera parte los puntos están distribuidos simétricamente. En la parte dos y tres, dentro de los cuadrados, además de los puntos, se hallan estímulos que visualmente son distractores. En la parte cuatro y cinco no existen distractores, pero los puntos se encuentran distribuidos de manera asimétrica. La prueba, de acuerdo a la tarea, presenta diferentes niveles de dificultad. Se instruye al sujeto para que colecte dos o más puntos con líneas rectas (en cada uno de los 35 cuadrados), de tal manera que la figura que elaboró en uno de los cuadros no se repita en los siguientes intentos (Lezak, 1995).

\section{Test de clasificación de tarjetas de Wisconsin}

Consiste en descubrir una regla o criterio de clasificación subyacente, a través del emparejamiento de una serie de tarjetas que varían en función de tres categorías básicas (color, forma y número).
Además, se debe adaptar la respuesta a los cambios en el criterio de clasificación, que se producen cada vez que el examinado da una serie de respuestas consecutivas correctas. El procedimiento de administración consiste en colocar frente al sujeto cuatro tarjetas alineadas horizontalmente. Luego, se le dan dos mazos de 64 cartas cada uno, y se le pide que empareje cada carta con las imágenes clave. El examinador proporciona una retroalimentación verbal cada vez que la persona responde, pero no revela la estrategia de clasificación necesaria, ni ofrece aclaraciones adicionales (Berg, 1948). Este instrumento cuenta con estudios en población infantil de habla hispana, en los que se ha incluido población colombiana (Roselli \& Ardila, 1993).

\section{Procedimiento}

Cada uno de los participantes fue evaluado de forma individual durante dos sesiones, con una duración de 60 minutos cada una; la mayoría de ellos fue valorada dentro de las instalaciones de la institución educativa a la que pertenecía y en la cual se garantizaba un espacio adecuado para la ejecución de la actividad. Todos los participantes en el estudio, firmaron un consentimiento informado, en donde se dieron a conocer las características del estudio.

\section{Análisis estadístico}

Para un diseño de corte transversal para dos grupos independientes y un nivel de correlación se hicieron comparaciones y se calcularon coeficientes de correlaciones estadísticas. Las correlaciones se realizaron entre las puntuaciones del CIV, CIT y CIE con la FE, en ambos grupos. Los datos fueron procesados en el paquete estadístico SPSS, versión 15. Dado que la mayoría de las variables estudiadas no cumplieron con el supuestos de distribución normal, de acuerdo con el test de contraste de Wilcoxon, se usó, para las comparaciones, la prueba no paramétrica U de Mann Whitney, y para las correlaciones, el coeficiente Rho de Spearman. 


\section{Resultados}

El estudio se realizó con niños y niñas pertenecientes a instituciones educativas privadas de la ciudad de Medellín, con edades comprendidas entre los 7 y 11 años, en el primero y segundo semestre de 2008.

Se evaluaron, inicialmente, 153 sujetos de una muestra preseleccionada por las instituciones, de los cuales 25 fueron excluidos por presentar criterios de trastorno por déficit de atención e hiperactividad (TDAH), de acuerdo con la información de los padres y los docentes; 58 , por obtener una puntuación en la CI por fuera del rango establecido para ambos grupos y 8 , por antecedentes de intervención psicológica debido a problemas emocionales o comportamentales.

La muestra estuvo conformada por 62 participantes, de los cuales 30 (52,9\% niños y 42,9\% niñas) pertenecían al grupo con capacidad intelectual promedio, con una edad media de 8.9 y una escolaridad de 3,2 ; los otros 32 sujetos $(47,1 \%$ niños y $57,1 \%$ niñas) fueron catalogados como talentos con una edad media de 9.1 y una escolaridad de 3.5. Ambos grupos pertenecían a los estratos socioeconómicos medio y alto.

En la Tabla 2, se observa que al comparar el rendimiento en las pruebas de función ejecutiva entre niños con talento y niños con inteligencia promedio, se encontraron, mediante la utilización de la prueba no paramétrica U de Mann Whitney, diferencias estadísticamente significativas $(p<$ $0,05)$ sólo en la prueba fluidez verbal fonológica.

En esta tabla se muestra que al calcular el tamaño del efecto, ninguna de las variables está igual o por encima 0,75 ; lo anterior indica que no existen diferencias clínicamente significativas entre los

TABLA 1

Características demográficas e intelectuales de los niños con talento y con inteligencia promedio

\begin{tabular}{|c|c|c|c|c|c|c|c|}
\hline \multirow[t]{2}{*}{ Variables } & \multicolumn{2}{|c|}{ Talentos $n: 32$} & \multicolumn{2}{|c|}{ Inteligencia promedio $\mathrm{n}: 30$} & \multicolumn{3}{|c|}{ U de Mann - Whitney } \\
\hline & Media & $D t^{*}$ & Media & $D t$ & $Z * *$ & & Valor $p$ \\
\hline Edad & 9.16 & 1.8 & 8.9 & 1,2 &,- 985 & &, 325 \\
\hline Escolaridad & 3.5 & 1.3 & 3,2 & 1,3 &,- 799 & & , 424 \\
\hline Sexo & $\mathrm{N}$ & $\%$ & $\mathrm{~N}$ & $\%$ & $\begin{array}{c}\text { Chi cuadrado } \\
, 625 \\
\end{array}$ & $\begin{array}{l}\mathrm{g} \\
1 \\
\end{array}$ & $\begin{array}{c}\text { Valor } p \\
, 429\end{array}$ \\
\hline Masculino & 16 & 47,1 & 18 & 52,9 & & & \\
\hline Femenino & 16 & 57,1 & 12 & 42,9 & & & \\
\hline Estrato & $\mathrm{N}$ & $\%$ & $\mathrm{~N}$ & $\%$ & 2,59 & 4 &, 627 \\
\hline Medio & 13 & 40.7 & 12 & 40 & & & \\
\hline Alto & 19 & 59.4 & 18 & 60 & & & \\
\hline \multirow{2}{*}{ Capacidad Intelectual } & \multirow{2}{*}{ Media } & \multirow{2}{*}{$D t$} & \multirow{2}{*}{ Media } & \multirow{2}{*}{$D t$} & \multicolumn{3}{|c|}{ U de Mann - Whitney } \\
\hline & & & & & Z & & Valor $p$ \\
\hline CI. verbal & 135,3 & 8.8 & 103,2 & 10,6 & $-6,645$ & &, 000 \\
\hline CI. ejecución & 135,8 & 9,8 & 100,1 & 8,8 & 6,766 & &, 000 \\
\hline CI. total & 138,6 & 1.3 & 101,8 & 5.7 & $-6,767$ & & ,000 \\
\hline
\end{tabular}

$n$ : Tamaño de la muestra * Dt: Desviación típica. ** Z: estadístico U de Mann- Whitney

Fuente: elaboración propia. Medellín 2008. 
TABLA 2

Comparación del desempeño en las pruebas de la FE en niños talento y niños con CI promedio

\begin{tabular}{|c|c|c|c|c|c|c|c|c|}
\hline \multirow{2}{*}{$\begin{array}{c}\text { Pruebas de Función } \\
\text { Ejecutiva }\end{array}$} & \multicolumn{2}{|c|}{ Talentos $(n: 32)$} & \multicolumn{2}{|c|}{ Inteligencia promedio $(n: 30)$} & \multicolumn{2}{|c|}{ U de Mann - Whitney } & \multicolumn{2}{|l|}{$\mathrm{TE}^{\mathrm{a}}$} \\
\hline & Media & $D t^{*}$ & Media & Dt & $2 * *$ & Valor $p$ & & \\
\hline $\begin{array}{l}\text { STROOP TEST Con- } \\
\text { flicto }\end{array}$ & 51,5 & 13,6 & 47,5 & 7,9 & -,861 & ,389 & 0,36 & \\
\hline $\begin{array}{l}\text { TORRE DE HANOI } \\
\text { Movimientos }\end{array}$ & 30 & 18,5 & 28,9 & 14,3 & -324 &, 746 & 0,07 & \\
\hline $\begin{array}{l}\text { Fluidez Verbal Fonoló- } \\
\text { gica }\end{array}$ & 22,4 & 8,6 & 17,4 & 6.8 & $-2,454$ &, $014^{*}$ & 0,65 & \\
\hline $\begin{array}{l}\text { Fluidez de diseños } \\
\text { (RUFFT) }\end{array}$ & 61,9 & 28.5 & 48.4 & 19,2 & $-1,937$ &, 053 & 0,55 & \\
\hline \multicolumn{9}{|c|}{ WISCONSIN CARD SORTING TEST } \\
\hline Categorías & 5.5 & 0.9 & 5,4 & 1.07 & &,- 706 & ,480 & 0,10 \\
\hline Errores perseverativos & 7,4 & 6,1 & 10,2 & 11,3 & &,- 226 & ,821 & 0,31 \\
\hline $\begin{array}{l}\text { Fallas para mantener el } \\
\text { principio }\end{array}$ & 0.88 & 1,3 & 1,4 & 1,7 & & $-1,516$ &, 129 & 0,35 \\
\hline
\end{tabular}

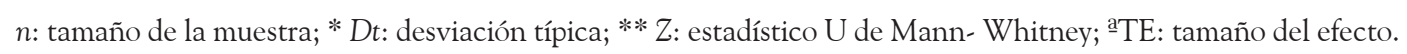

$* p<0.05$

Fuente: elaboración propia. Medellín 2008.

grupos. Sin embargo, se presenta un tamaño del efecto medio en las variables fluidez verbal fonológica $(0,65)$ y en fluidez de diseños $(0,55)$.

El análisis de correlación encontró sólo una correlación estadísticamente significativa, pero baja entre fluidez verbal fonológico y CI verbal, cuando se analizó la muestra en su conjunto.

\section{Discusión}

Los resultados mostraron que sólo la fluidez verbal fonológica diferenció los grupos de niños con talento de los niños con inteligencia promedio. Además, sólo hubo una correlación significativa, pero baja, entre el CI verbal y la fluidez fonológica cuando se analizó la muestra en su conjunto. Ardila et al. (2000) encontraron esta misma correlación baja entre la CI y las tareas de FE, en adolescentes de instituciones educativas públicas en Colom- bia. Este hallazgo podría ser interpretado, desde la perspectiva psicométrica, afirmando que las puntuaciones de los coeficientes intelectuales del WISC III no incluyen en su medición la evaluación de la FE. Esto corroboraría la afirmación de Ardila (1999) según la cual en la valoración de la capacidad intelectual, usando las pruebas disponibles en la actualidad, no se tiene en cuenta la inclusión de mediciones de las dimensiones de la FE, a pesar de ser un componente importante de la cognición humana y uno de los elementos fundamentales de la definición teórica de inteligencia.

Wechsler (2004), al definir la CI como "el agregado o la capacidad global del individuo para actuar hacia la consecución de un propósito, pensar racionalmente e interactuar efectivamente con su ambiente" (p. 26). Propuso cuatro elementos esenciales: 1) capacidad agregada o global, con la cual no se toma partido en la discusión de que 
TABLA 3

Correlaciones entre CI y FE en niños talentos (n: 32) y niños con inteligencia promedio (n: 30)

\begin{tabular}{lccccccc}
\hline & \multicolumn{2}{c}{ CI Total } & \multicolumn{2}{c}{ CI Verbal } & \multicolumn{2}{c}{ CI Ejecución } \\
\hline \multicolumn{1}{c}{ Función Ejecutiva } & Rho & Valor $\mathbf{p}$ & Rho & Valor $\mathbf{p}$ & Rho & Valor $\mathbf{p}$ \\
\hline Inhibición Stroop Test & 0,069 & 0,593 & 0,013 & 0,923 & 0,178 & 0,167 \\
Planeación ToH Movimientos &,- 023 &, 860 &, 004 &, 977 &,- 029 & 0,822 \\
Fluidez Fonológica & 0,231 & 0,071 & 0,267 & $0,036^{*}$ & 228 & 0,074 \\
Fluidez no Verbal (Ruff) & 0,179 & 0,164 & 0,226 & 0,077 & 0,126 & 0,331 \\
Selección Wcst Categorías & 0,071 & 0,581 & 0,043 & 0,740 & 0,095 & 0,462 \\
Monitoreo WCST Errores Perseverativos &,- 086 &, 508 &,- 002 &, 987 & $-0,124$ & 0,338 \\
Monitoreo WCST Fallas Para Mantener El Principio & $-0,146$ & 0,256 & $-0,150$ & 0,244 & $-0,155$ & 0,229 \\
\hline
\end{tabular}

$n$ : tamaño de la muestra

*La correlación es significativa al nivel 0,05 (bilateral).

${ }^{\circ}$ Rho: coeficiente de correlación de Spearman

Fuente: elaboración propia. Medellín 2008.

es un factor $(\mathrm{g})$ o que es el conjunto habilidades cognitivas; 2) el actuar con propósito: se refiere a la organización y planeación, es decir pura función del lóbulo Frontal; 3) es pensar racionalmente, el cual hace referencia a la función ejecutiva, es decir requiere de metacognición, resolución de problemas, control de la cognición, organización y planeación de la conducta; y, 4) es adaptarse efectivamente al entorno, lo cual quiere decir que para hablar de inteligencia, se requiere que sea contextualizada. Sin embargo, a pesar de estar la FE plasmada en su definición de CI, no es evaluada por sus escalas (Ardila, 1999).

La inteligencia general o factor $\mathrm{g}$, la inteligencia cristalizada (CIV) -la cual tiene que ver con todos los conocimientos del mundo, la experiencia y el aprendizaje - y la inteligencia fluida (CIP) capacidad de razonamiento, de invención, de creación de nuevos conceptos y establecer relaciones nuevas, ligada al desarrollo neurológico, libre de las influencias culturales o sociales (Carroll, 1993), según los resultados del presente estudio, no mostraron una correlación fuerte con ninguna de las dimensiones del subsistema comparador de la FE (inhibición, planeación, fluidez verbal fonológica, fluidez no verbal, monitoreo y selección de objetivos), de acuerdo con el modelo teórico trabajado.

Al comparar el rendimiento de los niños con inteligencia promedio y los niños con talento en las tareas de FE, no se encontraron diferencias significativas entre los grupos con diferentes niveles de inteligencia general, lo cual no apoyaría la hipótesis de la existencia de unos niveles más altos de organización, planeación y monitoreo en los niños con una CI superior. Esto indicaría que la CI, medida con el CIT de un individuo, no dependería de la capacidad de su FE, medida con las pruebas estandarizadas tradicionales. Estos mismos resultados han sido referenciados por otras investigaciones (Donders \& Kirsh, 1991; Johnstone, Holland \& Larimore, 2000; Welsh et al., 1991).

De acuerdo con lo anterior, la presente investigación confirma los hallazgos de Welsh y et al. (1991) quienes no encontraron correlación entre CI y FE en niños de 6 a 12 años de edad. Así mismo se corrobora que las dimensiones de la FE en el desarrollo normal humano, son relativamente independientes de las mediciones de la CI obtenidas con las pruebas tradicionales de inteligencia general (Donders \& Kirsh, 1991; Johnstone et al., 2000). 
Uno de los supuestos que se podría sustentar con los resultados sería que hay ausencia relativa de componentes de la FE en la administración de tests clásicos de inteligencia, lo cual ha sido descrito también por otros autores (Denckla, 1996; Stuss, 1992; Welsh et al., 1991). Las versiones recientes de las escalas de inteligencia, entre ellas el WISC III (Wechsler, 2004), hicieron un esfuerzo por tratar de corregir el error de la ausencia de elementos que midieran la FE. Para ello, introdujeron tareas como: construcción con cubos, ordenamiento de historietas y laberintos. Los resultados sugieren que estas modificaciones no corrigieron de manera adecuada las fallas en la medición de la FE, encontradas en las versiones anteriores de la escala de esta escala de inteligencia. Esto significa que la aplicación del WISC III no es suficiente para medir la generación de patrones de resolución de problemas novedosos, el mantenimiento de estos patrones a través del tiempo o la necesidad de manipularlos de forma flexible cuando las circunstancias son cambiantes, por lo cual se necesitaría la aplicación de tareas específicas en la medición de estas dimensiones (Ardila, 1999).

Los datos también pudieran interpretarse de acuerdo con las explicaciones clínicas de Damasio y Anderson (1993), quienes señalaron que la afectación de la función ejecutiva por lesiones del lóbulo frontal, no altera la capacidad intelectual general del individuo, cuando es medida con las pruebas estandarizadas que calculan el factor $\mathrm{g}$. La corteza prefrontal, de acuerdo con este modelo, estaría integrada a tres circuitos independientes de control y supervisión: el dorsolateral (FE cognitiva), orbitofrontal (control emocional) y medial (control conductual y atencional). Las alteraciones propias de cada síndrome prefrontal dependerían del circuito afectado por la lesión. Las pruebas de inteligencia general miden muy tangencialmente las alteraciones cognitivas de la FE, pero no evalúan los niveles de los trastornos emocionales, conductuales y atencionales relacionados con las actividades de organización y autosupervisión de los lóbulos frontales.

De otro lado, en las pruebas específicas estandarizadas de medición de la FE, en la investigación actual se encontró que en los niños con talento y los niños con $\mathrm{CI}$ promedio tuvieron ejecuciones similares, lo que difiere de lo informado por Arffa y et al. (1998) quienes encontraron que niños y jóvenes con mayor CI presentan un mejor rendimiento en el WCST que los niños con CI mediaalta. Sin embargo, al revisar los datos publicados por este estudio se encontró que los autores realizaron el análisis comparativo entre los grupos con puntuaciones normativas del WCST extraídos de otros estudios, con poblaciones de características diferentes. También se observó que estos autores derivaron las conclusiones a partir de análisis de varianza multivariado con grupos de 10 individuos, usando incluso un análisis de regresión escalonado, procedimiento que no es adecuado para el tamaño de la muestra; por lo tanto, los resultados podrían representar un ruido estadístico, generado por los procedimientos usados para los análisis. En la publicación de los datos sólo se informaron los promedios y se omitieron las desviaciones estándar, por lo que no es posible calcular el tamaño del efecto, para determinar la significación clínica de los datos, ni el grado de dispersión encontrado.

En conclusión, los resultados obtenidos por los dos grupos en la ejecución de las pruebas que evalúan la FE, no permiten establecer diferencias ni estadísticas ni clínicamente significativas entre el grupo niños con talento y niños con inteligencia promedio. La única dimensión de la FE con una correlación significativa $(p<0,036)$ pero baja $(r$ $=0,267$ ), con alguna de las medidas de la CI, fue fluidez verbal fonológica, que se correlacionó con el CI verbal. Se asume que la correlación esta dada por la carga lingüística de las tareas, la significancia estadística no implica que esta correlación sea importante, sino que, dado el tamaño de la muestra y el diseño usado, se descarta el efecto del azar. Esto llevaría a rechazar la hipótesis de trabajo, según la cual existiría una alta correlación entre la CI y la FE, especialmente en la organización y flexibilidad cognitivas, medidas con el WCST, con una alta producción en tareas de fluidez verbal controladas (Arffa, 2007). 
- Se aceptan, como limitaciones del estudio, el nivel de generalización de los datos y la ausencia de estandarización de la prueba de capacidad intelectual WISC III, en población colombiana.

Se recomienda para futuros estudios:

- Incluir niños con condiciones socioeconómicas bajas y/o pertenecientes a instituciones educativas públicas;

- Realizar un análisis correlacional entre la función ejecutiva y los factores derivados de la medida de la capacidad intelectual con el WISC III, tales como: comprensión verbal, organización perceptual, ausencia de distraibilidad y velocidad de procesamiento;

- Elaborar estudios en el que se analice el rendimiento en pruebas de FE en diferentes grupos etarios con características de normalidad y con coeficientes intelectuales diferentes (inteligencia limítrofe, debajo del promedio, dentro del promedio, alta, superior, y muy superior).

Esto, con el fin de determinar definitivamente que la dimensiones capacidad intelectual y de la función ejecutiva son factores independientes.

\section{Referencias}

Álvarez, L. M. (2006). La torre de Hanoi como instrumento de evaluación cognoscitiva en una muestra de niños en edad escolar. Tesis de doctorado no publicada, Recinto de Rio Piedras, Universidad de Puerto Rico.

Arango-Tobón, O. E. \& Puerta-Lopera, I. C. (2008). El papel de la función ejecutiva en la conducta, la cognición y la emoción. En D. A. Pineda (Ed.), 10 años de investigación en neuropsicología (pp. 65-80). Medellín: Editorial Universidad de San Buenaventura.

Ardila, A. (1999). Neuropsychological approach to intelligence. Neuropsychological Review, 9, 117-136.

Ardila, A., Pineda, D. \& Rosselli, M. (2000). Correlation between intelligence test scores and executive function measures. Archives of Clinical Neuropsychology, 15, 31-36.
Ardila, A., Rosselli, M. \& Puente, M. (1994). Neuropsychological assessment of Spanish speaker. New York: Plenum Press.

Arffa, S. (2007). The relationship of intelligence to executive function and non-executive function measures in a sample of average, above average, and gifted youth. Archives of Clinical Neuropsycho$\log$, 22, 969-978.

Arffa, S., Lovell, M., Podell, K. \& Goldberg, E. (1998). Wisconsin card sorting test performance in above average and superior school children: Relationship to intelligence and age. Archives of Clinical Neuropsychology, 13, 713-720.

Berg, E. A. (1948). A simple objective test for measuring flexibility in thinking. Journal of General Psychology, $39,15-22$.

Blair, C. (2006). How similar are fluid cognition and general intelligence? A developmental neuroscience perspective on fluid cognition as an aspect of human cognitive ability. The Behavioral and brain sciences, 29, 109-125.

Cattel, R. B. (1963). Theory of fluid and cristalized intelligence: A critical experiment. Journal of Educational Psychology, 54, 1-22.

Carroll, J. B. (1993). Human CognitiveAbilities: A survey of factor Analytic Studies. Cambridge : Cambridge University Press.

Carroll, J. B. (1994). Constructing a Theory from data. En D. K. Detterman (Ed.), Current topics in Human Intelligence. Theories of intelligence (Vol. 4, pp. 43-64). Nor Wood, NJ: Ablex Publishing Corporation.

Damasio, A. \& Anderson, S. (1993). The frontal lobes. In K. M. Heilman \& E. Valestein (Eds.), Clinical Neuropsychology (3rd ed., pp 409-460). New York: Oxford University Press.

Denckla, M. B. (1996). Research on executive function in a neurodevelopmental context: Application of clinical measures. Developmental Neuropsychology 12, 5-15.

Donders, J. \& Kirsch, N. (1991). Nature and implications of selective impairment on the Blooklet Category Test and Wisconsin card Sorting Test. Clinical Neuropsychology, 5 (1), 78-82.

Friedman, N., Miyake, A., Corley, R. P., Young, S. E., DeFries, J. C. \& Hewitt, J. K. (2006). Not all 
executive functions are related to intelligence. Psychological Science, 17 (2), 172.

García-Barrera, M. (2008). Alteraciones de la función ejecutiva como factor común a los trastornos del comportamiento en los niños escolarizados. En D. A. Pineda (Ed.), 10 años de investigación en neuropsicología (pp. 65-80). Medellín: Editorial Universidad de San Buenaventura.

Gioia, G. A., Isquith, P. K., Retzlaff, P. D. \& Espy, K. A. (2002). Confirmatory factor analysis of the Behavior Rating Inventory of Executive Function (BRIEF) in a clinical sample. Child Neuropsycho$\log , 8,249-257$.

Golden, C. J. (1981). The Luria Nebraska children's battery: Theory and formulation. In G. W. Hynd \& J. E. Obrzut (Eds.), Neuropsychological assessmente and the school aged child (pp. 277-302). New York: Grune \& Strattion.

Guilford, J. P. (1985). The structure of intellect model. En B. B. Wolman (Ed.), Handbook of Intelligence: Theories, measurements, and applications. New York: Wiley.

Johnstone, B., Holland, D. \& Larimore, C. (2000). Language and academic abilities. In G. Groth-Marnat (Ed.), Neuropsychological Assessment. In Clinical Practice: A guide to test interpretation (pp. 335-354). New York: John Wiley \& Sons, Inc.

Lezak, M. D. (1995). Neuropsychological Assessment (3rd ed.). New York: Oxford University Press.
Mark, E., Hagelthorn, K. M., Cutting, L. E., Schuerholz, L. J., Pelletier, S. F., Rawlins, C. et al. (2002). Effects of IQ on executive function measures in children with ADHD. Child Neuropsychology, 8 (1), 52-65.

Milner, B (1982). Some cognitive effects of frontal-lobe lesions in man. Philosophical Transactions of the Royal Society of London, 298, 211-226.

Passow, A. (1993). National/State policies regarding education of the gifted. En K. Heller, F. Mönks \& A. Passow (Eds.), International Handbook of Research and Development of Giftedness and Talent (pp. 29-46). Oxford: Pergamon Press.

Spearman, C. (1923). The nature of Intellicence and the principles of Cognition. London: Macmillan.

Stuss, D. T. (1992). Biological and psychological development of executive functions. Brain and Cognition, $20,8-23$.

Thurstone, L. L. (1947). Multiple Factor Analysis. Chicago: University of Chicago Press.

Wechsler, D. (2004). Test de Inteligencia para niños WISC III: manual. Buenos Aires: Paidós.

Welsh, M. C., Pennington, B. F. \& Groisser, D. B. (1991). A normative-developmental study of excutive function: A window on prefrontal function in children. Developmental Neuropsychology, 7 (2), 131-149. 
